\title{
Testing Topology Conserving Gauge Actions for Lattice QCD
}

\section{Kei-ichi Nagai*, Karl Jansen}

John von Neumann-Institute für Computing NIC,

DESY, Platanenallee 6, D-15738 Zeuthen, Germany

Email: Keiichi.Nagai@desy.de, Karl.Jansen@desy.de

\section{Wolfgang Bietenholz, Luigi Scorzato}

Institut für Physik, Humboldt Universität zu Berlin

Newtonstr. 15, D-12489 Berlin, Germany

Email: bietenho@physik.hu-berlin.de,

Luigi.Scorzato@physik.hu-berlin.de

\section{Silvia Necco}

Centre de Physique Théorique, Luminy, Case 907

F-13288, Marseille Cedex 9, France

Email: heccodcpt.univ-mrs.fr

\section{Stanislav Shcheredin}

Fakultät für Physik, Universität Bielefeld

D-33615 Bielefeld, Germany

Email: shchered@physik.hu-berlin.de

\begin{abstract}
We explore gauge actions for lattice QCD, which are constructed such that the occurrence of small plaquette values is strongly suppressed. Such actions originate from the admissibility condition in order to conserve the topological charge. The suppression of small plaquette values is expected to be advantageous for numerical studies in the $\varepsilon$-regime and also for simulations with dynamical quarks. Performing simulations at a lattice spacing of about $0.1 \mathrm{fm}$, we present numerical results for the static potential, the physical scale $r_{0}$, the stability of the topological charge history, the condition number of the kernel of the overlap operator and the acceptance rate against the step size in local HMC algorithm.
\end{abstract}

XXIIIrd International Symposium on Lattice Field Theory

25-30 July 2005

Trinity College, Dublin, Ireland

\footnotetext{
* Speaker.
} 


\section{Introduction and motivation}

Chiral perturbation theory $(\chi \mathrm{PT})$ [1] and lattice QCD are powerful tools to extract quantities at low energy which are relevant for QCD. Lattice QCD simulations can provide the determination of the Low Energy Constants (LECs) of $\chi \mathrm{PT}$ from first principles calculations. A particular situation is found when one enters the $\varepsilon$-regime [2], where

$$
m_{\pi} \sim \frac{1}{L^{2}} \quad, \quad m_{\pi}^{-1}>L \quad, \quad\left(L \gg \frac{1}{2 F_{\pi}}\right) .
$$

There are analytical formulae from $\chi \mathrm{PT}$ that describe the behaviour of physical quantities in the $\varepsilon$-regime as a function of the volume and the quark mass. These formulae are parametrized by the infinite volume LECs of the effective chiral Lagrangian. Therefore we can extract physically relevant information even from the unphysical $\varepsilon$-regime. As a peculiarity of the $\varepsilon$-regime, since one is working in small boxes $(1.1 \mathrm{fm} \cdots 1.5 \mathrm{fm})$, observables depend significantly on the topological sector, and predictions exist for expectation values in specific sectors [3]. For the parameters that have been used in the $\varepsilon$-regime simulations, it would be of particular interest to collect large sets of configurations ${ }^{1}$ with the index $|v|>0$.

In general, however, it is not obvious to define topological sectors on the lattice. A neat definition exists with overlap fermions satisfying the Ginsparg-Wilson relation [5, 6]

$$
D_{\mathrm{ov}}^{(0)} \gamma_{5}+\gamma_{5} D_{\mathrm{ov}}^{(0)}=\frac{1}{\mu} D_{\mathrm{ov}}^{(0)} \gamma_{5} D_{\mathrm{ov}}^{(0)}, \quad D_{\mathrm{ov}}^{(0)}=\mu\left[1+\gamma_{5} Q / \sqrt{Q^{2}}\right], \quad Q=\gamma_{5}\left(D_{\mathrm{W}}-\mu\right),
$$

where $\mu \gtrsim 1$ is a mass parameter in the operator $Q$. Since $D_{\mathrm{ov}}^{(0)}$ has exact zero modes with definite chiralities [6, 7, 8], therefore one can use the index as a definition of the topological charge due to the Atiyah-Singer theorem.

While overlap fermions define the fermion-sector of lattice $\mathrm{QCD}$, if one insists on exact lattice chiral symmetry, the lattice gauge action of QCD can be constructed in many ways [- [7]. One requires the naive continuum limit for all lattice gauge actions to coincide, in which case they fall into the same universality class. The simplest formulation is the Wilson plaquette action

$$
S_{\mathrm{W}}[U]=\beta \sum_{P} S_{P}\left(U_{P}\right), \quad S_{P}\left(U_{P}\right)=1-\frac{1}{3} \operatorname{Re} \operatorname{Tr} U_{P},
$$

where the sum over $P$ runs over all plaquettes.

For the overlap operator (1.2), the topological transitions are excluded under continuous deformations if all the plaquette variables $U_{P}$ in the configurations involved obey the inequality [10, 11] $S_{P}\left(U_{P}\right)<\varepsilon=\frac{2}{5 d(d-1)}=\frac{1}{30}$ (for all $P$ ). Later on H. Neuberger showed a more tolerant bound [12] $\varepsilon=\frac{1}{(1+1 / \sqrt{2}) d(d-1)} \simeq \frac{1}{20.5}$. For this admissibility condition, the exponential locality of the GinspargWilson fermions is guaranteed [11], and the topological charge is conserved rigorously. However, it is very difficult to realize this condition in the numerical simulation. For practical purposes we have to relax $\varepsilon$ to larger values than the analytical bounds.

\footnotetext{
${ }^{1}$ The topologically neutral sector is problematic due to the frequent appearance of very small Dirac eigenvalues, which leads to strong spikes in the Monte Carlo histories of correlation functions [ $[$ ].
} 


\section{Proposal of the gauge actions}

We now describe a number of non-standard lattice gauge actions, which suppress the unwanted small plaquette values leading to transitions between different topological sectors. The naive continuum limit of these actions coincides with that of Wilson action (1.3). Based on the proposal by M. Lüscher [10], we consider topology conserving gauge actions [14]

$$
\begin{array}{ll}
S_{\varepsilon, n}^{\text {hyp }}\left(U_{P}\right) & =\frac{S_{P}\left(U_{P}\right)}{\left[1-S_{P}\left(U_{P}\right) / \varepsilon\right]^{n}} \quad \text { for } S_{P}\left(U_{P}\right)<\varepsilon \quad \text { and } \quad+\infty \text { otherwise }, \\
S_{\varepsilon, n}^{\text {pow }}\left(U_{P}\right) & =S_{P}\left(U_{P}\right)+\frac{1}{\varepsilon} S_{P}\left(U_{P}\right)^{n}, \\
S_{\varepsilon, n}^{\exp }\left(U_{P}\right) & =S_{P}\left(U_{P}\right) \cdot \exp \left\{S_{P}\left(U_{P}\right)^{n} / \varepsilon\right\},
\end{array}
$$

In simulations, the action (2.1) with $n=1$ was first used in the Schwinger model [13]. They set $\varepsilon=1$, i.e. far above the theoretical value of about 0.29 , but they still observed topological stability over hundreds of configurations. Here we investigate this type and its extension to the gauge action of lattice QCD.

We show the basic properties of these actions. The left plot of fig. 1 is the histogram of plaquette values for the action $S_{\varepsilon, n=1}^{\text {hyp }}$, compared to the Wilson action, on a $4^{4}$ lattice. In the topology conserving gauge action, the occurrence of very small plaquette values is drastically suppressed. The right plot of fig. 1 shows the ratio of the force in the HMC algorithm between the Wilson plaquette action $S_{P}$ and topology conserving gauge actions. The action $S_{\mathcal{E}, n}^{\exp }$ makes a sharp wall continuous, but shows the same behaviour as the Wilson action over a wide range.
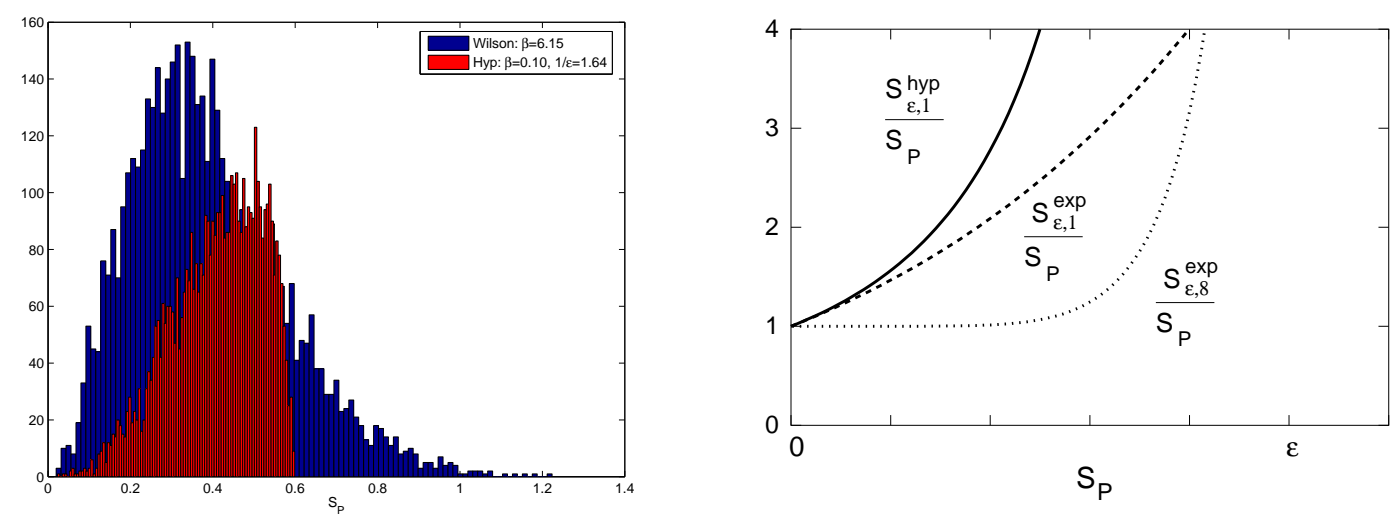

Figure 1: Left: Histograms of $S_{P}$ for the topology conserving gauge action, compared to the Wilson action, on a $4^{4}$ lattice. Right: Ratio of the force between the Wilson action $S_{P}$ and modifi ed actions.

For the generation of the configurations we used a local HMC algorithm. Since $S_{\mathcal{\varepsilon}, n}^{\text {hyp }}$ is nonlinear for the link variables, the heatbath and over-relaxation algorithms are not straightforwardly applicable. We use a $16^{4}$ lattice and measure physical quantities every 50 trajectories. See table 1 about the acceptance rate of local HMC. In the value $1 / \varepsilon=1.64$, the acceptance rate is about $65 \%$ at the Molecular dynamics step $d \tau=0.1$. However the acceptance becomes good as $d \tau$ decreases. 

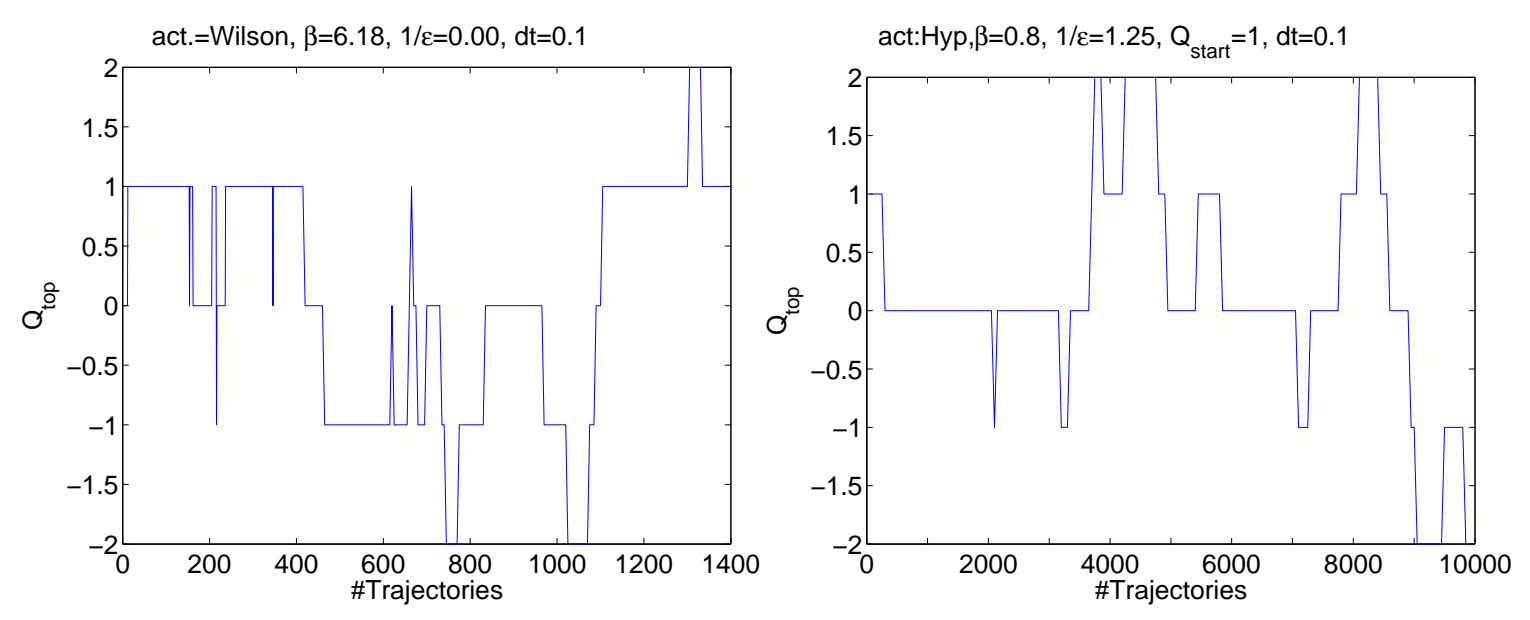

Figure 2: MC-history of $Q_{t o p}$ for various types of the gauge actions.

\section{Monte Carlo history of $Q_{t o p}$ : Stability of the topological charge.}

In fig. 2 we show the Monte Carlo history of the topological charge $Q_{t o p}$, which is evaluated by the cooling method ${ }^{2}$, for the Wilson action and the modified gauge actions. The topological charge in $1 / \varepsilon \ll 20.5$ is not always conserved. However, the changes of $Q_{t o p}$ are suppressed. Here we define the quantity representing the stability of the topological charge: $f_{\text {top }}=$ (the number of jumps of $Q_{t o p}$ )/(the number of trajectories), see also table 1. The value $f_{\text {top }}$ for the modified gauge actions is 10 times smaller than for the Wilson action and thus these actions clearly stabilize the topology charge. Also the autocorrelation of the plaquette $\tau^{\text {plaq }}$ is getting shorter as $1 / \varepsilon$ increases. This is important and typical properties of topology conserving gauge actions.

\section{The static potential, the physical scale and lattice artifacts}

In this section we evaluate the physical scale and the lattice artifacts for various parameters of $\beta$ and $1 / \varepsilon$ in the modified gauge actions. The most established method of setting a scale in pure gauge theory is the measurement of the static potential and the force at relatively large distances, extracted from Wilson loops and see e.g. Ref. [15].

We show the left plot of fig. 3. A way to check the lattice artifacts [15] is to compare the short distance force at finite lattice spacing with the one extrapolated to the continuum limit $\left.r^{2} F\left(r / r_{0}\right)\right|_{c}$

$$
\Delta\left(r / r_{0}\right)=\frac{r^{2} F\left(r / r_{0}\right)-\left.r^{2} F\left(r / r_{0}\right)\right|_{c}}{\left.r^{2} F\left(r / r_{0}\right)\right|_{c}} .
$$

As seen in this figure, for increasing $1 / \varepsilon$, the discretization errors increase as well. Typically the artifacts of $S_{\varepsilon, n=1}^{\text {hyp }}$ at $1 / \varepsilon \gtrsim 1$ are about $10 \%$. Even in $1 / \varepsilon=1.64$, the lattice artifact is less than $15 \%$. This is comparable with the Iwasaki and DBW2 gauge actions. Note that the static potential at distance $r \sim r_{0}$ has not been determined with high precision, and this is reflected in large uncertainties on the quantities $\Delta\left(r / r_{0}\right)$.

\footnotetext{
${ }^{2}$ The cooling charges agree in practically all cases with the overlap indices in the range $\mu=1.3, \ldots, 1.6$, as we tested for a subset of the confi gurations.
} 

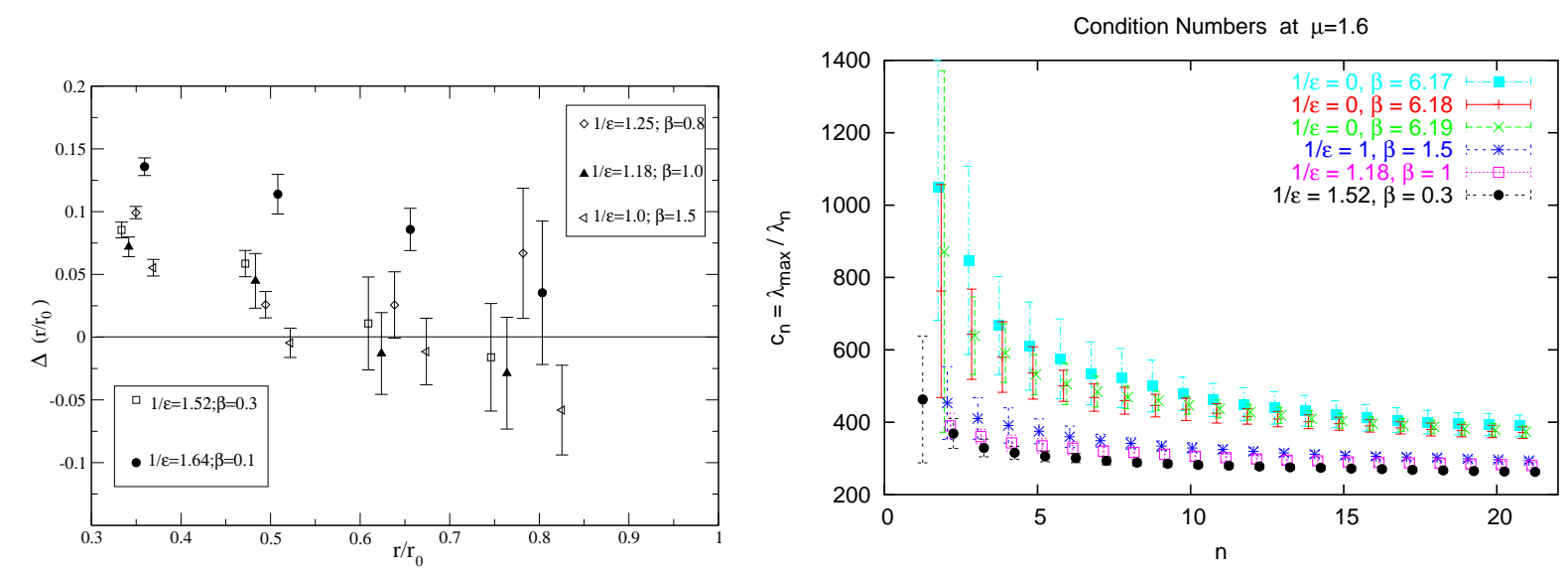

Figure 3: Left: Lattice artifact by the standard estimate for the action $S_{\varepsilon, n=1}^{\text {hyp }}$. Right: The conditions numbers $c_{2} \ldots c_{21}$ at $\mu=1.6$.

\section{Condition number for the overlap operator}

We also evaluate the effect of topology conserving gauge actions on the condition number of the overlap operator (1.2). We observe the condition number defined as $C_{n}=\frac{\lambda_{\max }}{\lambda_{n}}$ where $\lambda_{\max }$ is the maximum eigenvalue and $\lambda_{n}$ is the $n$ 'th eigenvalue of the kernel $Q^{2}$ in the overlap operator. $C_{n}$ is relevant if $n-1$ modes of $Q^{2}$ are projected out. Fig. 3 (right plot) shows the behaviour of the condition number $c_{2}, \cdots, c_{21}$ at $\mu=1.6$ and about $r_{0} / a \sim 7.0$. We see an improvement which increases if only a few modes are projected out and if $1 / \varepsilon$ grows. This means that the numerical computation of $Q / \sqrt{Q^{2}}$ in the overlap operator gets fast by using topology conserving gauge actions.

\section{Summary}

Simulations in the $\varepsilon$-regime of chiral perturbation theory using the overlap operator can be simplified when appropriate choices of the gauge actions are made. We tested a number of gauge actions that suppress small plaquette values and investigated the properties of these actions [16]. Our results can be summarized as follows, see also table 1 .

- The actions of eqs. 2.1, 2.2, 2.3) stabilize the topological charge and could therefore be profitable in QCD simulations in the $\varepsilon$-regime.

- The actions of eqs. (2.2, 2.3) are conceptually clean, have a positive transfer matrix and show lattice artifacts that are acceptable and compare well with those of the Iwasaki and DBW2 gauge actions.

- The condition number of the kernel of the overlap operator is improved by using the topology conserving gauge actions when compared to the standard Wilson plaquette action. This speeds up the simulations of overlap fermions in the quenched approximation and will also help in dynamical simulations using the (global) HMC algorithm. 


\begin{tabular}{|c|c|c|c|c|c|c|c|}
\hline$\varepsilon^{-1}$ & $\beta$ & $r_{0} / a$ & $\beta_{W}$ & $d \tau$ & $\tau^{\text {plaq }}$ & $f_{\text {top }}$ & acc. rate \\
\hline \hline 0. & 6.19 & $7.14(3)$ & 6.19 & 0.1 & $7(1)$ & $2.2 \mathrm{e}-2$ & $>99 \%$ \\
\hline 1. & 1.5 & $6.6(2)$ & $6.13(2)$ & 0.1 & $2.2(1)$ & $2.4 \mathrm{e}-3$ & $>99 \%$ \\
1. & 1.5 & $6.6(2)$ & $6.13(2)$ & 0.01 & $2.2(1)$ & $3.2 \mathrm{e}-3$ & $>99 \%$ \\
\hline 1.18 & 1. & $7.2(2)$ & $6.18(2)$ & 0.1 & $1.2(1)$ & $1.6 \mathrm{e}-3$ & $>99 \%$ \\
1.18 & 1. & $7.2(2)$ & $6.18(2)$ & $0.02 / 0.01$ & $1.3(1)$ & $1.4 \mathrm{e}-3$ & $>99 \%$ \\
\hline 1.25 & 0.8 & $7.0(1)$ & $6.17(1)$ & 0.1 & $1.1(1)$ & $2.5 \mathrm{e}-3$ & $>99 \%$ \\
\hline 1.52 & 0.3 & $7.3(4)$ & $6.19(4)$ & 0.1 & $0.8(1)$ & $9.4 \mathrm{e}-4$ & $\approx 95 \%$ \\
\hline 1.64 & 0.1 & $6.8(3)$ & $6.15(3)$ & 0.1 & $1.0(1)$ & $7.0 \mathrm{e}-4$ & $\approx 65 \%$ \\
1.64 & 0.1 & & & 0.05 & $0.7(1)$ & $2.3 \mathrm{e}-3$ & $\approx 78 \%$ \\
1.64 & 0.1 & & & 0.025 & $0.6(1)$ & $3.5 \mathrm{e}-3$ & $\approx 93 \%$ \\
\hline
\end{tabular}

Table 1: Results for $S_{\varepsilon, 1}^{\text {hyp }}$ for various values of $\varepsilon$ and $\beta$, on a $16^{4}$ lattice. $\beta_{W}$ is the corresponding $\beta$ of Wilson action to the obtained $r_{0} / a$. $\tau^{\text {plaq }}$ is the autocorrelation time of the plaquette value.

\section{References}

[1] S. Weinberg, Physica A 96 (1979) 327; J. Gasser and H. Leutwyler, Ann. Phys. 158 (1984) 142.

[2] J. Gasser and H. Leutwyler, Phys. Lett. B 188 (1987) 477.

[3] H. Leutwyler and A. Smilga, Phys. Rev. D 46 (1992) 5607.

[4] W. Bietenholz, T. Chiarappa, K. Jansen, K.-I. Nagai and S. Shcheredin, JHEP 0402 (2004) 023 [hep-lat/0311012].

[5] P.H. Ginsparg and K.G. Wilson, Phys. Rev. D 25 (1982) 2649.

[6] H. Neuberger, Phys. Lett. B 417 (1998) 141 [hep-lat/9707022].

[7] P. Hasenfratz, V. Laliena and F. Niedermayer, Phys. Lett. B 427 (1998) 125 [hep-1at/9801021]; P. Hasenfratz, Nucl. Phys. B 525 (1998) 401 [hep-lat/9802007].

[8] M. Lüscher, Phys. Lett. B 428 (1998) 342 [hep-lat/9802011].

[9] K.G. Wilson, Phys. Rev. D 10 (1974) 2445; M. Lüscher and P. Weisz, Phys. Lett. B 158 (1985) 250, Commun. Math. Phys. 97 (1985) 59; Y. Iwasaki, UTHEP-118, (1983), unpublished; QCDTARO Collaboration (Ph. de Forcrand et al.), Nucl. Phys. (Proc. Suppl.) B 53 (1997) 938 [hep-lat/9608094]; T. Takaishi, Phys. Rev. D 54 (1996) 1050.

[10] M. Lüscher, Nucl. Phys. B 549 (1999) 295 [hep-lat/9811032].

[11] P. Hernández, K. Jansen and M. Lüscher, Nucl. Phys. B 552 (1999) 363 [hep-lat/9808010].

[12] H. Neuberger, Phys. Rev. D 61 (2000) 085015 [hep-lat/9911004].

[13] H. Fukaya and T. Onogi, Phys. Rev. D 68 (2003) 074503 [hep-lat/ 0305004 ], Phys. Rev. D 70 (2004) 054508 [hep-lat/ 0403024$].$

[14] W. Bietenholz, K. Jansen, K.-I. Nagai, S. Necco, L. Scorzato and S. Shcheredin, [hep-lat/0409073, hep-lat/0412017].

[15] S. Necco, Ph.D. thesis (Berlin, 2004) [hep-lat/0306005], Refereces therein.

[16] W. Bietenholz, K. Jansen, K.-I. Nagai, S. Necco, L. Scorzato and S. Shcheredin, in preparation. 